\title{
Higher Order Structure and Thermo-Responsive Properties of Polymeric Gel with Crystalline Side Chains
}

\author{
Xiaowei He, Yushi Oishi, ${ }^{\dagger}$ Atsushi Takahara, and Tisato KaJiYama* \\ Department of Chemical Science \& Technology, Faculty of Engineering, Kyushu University, \\ Hakozaki, Higashi-ku, Fukuoka 812-81, Japan
}

(Received November 8, 1995)

\begin{abstract}
Crosslinked polymeric gel films were prepared by a radical copolymerization of stearyl acrylate (SA) and acrylic acid (AA) with $N, N^{\prime}$-methylenebisacrylamide (MBAA) as a cross-linker. Aggregation structure of the (SA/AA/MBAA, 24.7/74.3/1.0; molar ratio) gel film was investigated on the basis of X-ray diffraction study. The gel films in both a dried and a swollen states in dimethyl sulfoxide (DMSO) formed crystalline lamellar structure at room temperature owing to intermolecular aggregation of stearyl acrylate side chains. The thermo-responsive behavior of the (SA/AA/MBAA) gel film was examined by the temperature dependence of the swelling ratio. The swelling ratio of the (SA/AA/MBAA, 24.7/74.3/1.0) gel film increased with an increase in temperature up to $320 \mathrm{~K}$ (the melting temperature of stearyl acrylate side chain crystals), whereas it decreased above $320 \mathrm{~K}$. In order to improve the thermo-responsive behavior, the stretched gel film was prepared by uniaxial stretching. The magnitude of melting endotherm for the stretched (SA/AA/MBAA) gel film increased with the stretching ratio and also, the crystallinity and/or the regularity of layer structure of the stretched gel film was improved by stretching, i.e. the lamellar long axis in the stretched gel aligned along the stretching direction. The thermo-responsive properties of the stretched gel films were also examined by the temperature dependence of the swelling ratio and the length of the gel film. The stretched gel film abruptly shrinked to the original length upon heating at melting temperature of stearyl acrylate side chain crystals. KEY WORDS Crosslinked Polymeric Gel / Higher-Order Structure / Thermo-Responsive Property Side Chain Crystal / Structural Control / Shape-Memory Effect / Stearyl Acrylate / Wide-Angle X-Ray Diffraction / Small-Angle X-Ray Scattering /
\end{abstract}

Polymeric gels which show responsive characteristics to external stimulus have been applied for various functions such as artificial muscle, drug delivery system and so on. ${ }^{1,2}$ Copolymerization/crosslinking of monofunctional and multifunctional monomers has been extensively used to prepare responsive gels of vinyl monomers, especially of a wide variety of acrylamide derivatives such as $\mathrm{N}$ isopropylacrylamide, and diethylacrylamide. ${ }^{3-5}$ These cross-linked gels of synthetic polymer usually exhibited amorphous structure and did not show any macroscopic and microscopic orders. On the other hand, it is known that biopolymer gels with ordered structure such as actin and myosin gels (the principal protein component of muscle) have various excellent properties. ${ }^{6-8}$ Then, if synthetic polymeric gels with order structure or mechanically and optically anisotropic characteristics materials were prepared, a novel material like to biopolymer gels can be expected.

Although the properties of synthetic gels have been the subject of a great deal of works, ${ }^{9,10}$ the structural control of polymeric gels based on the order structure has not been studied extensively. Osada and co-workers prepared the crystalline polymeric gel by copolymerization of an acrylic monomer with hydrophobic long alkyl side chains. ${ }^{11,12}$ They have investigated the effect of monomer composition on the ordered structure of the gel, and the aggregation structure in the gel was compared with those in the un-crosslinked polymers. Their differential scanning calorimetric and polarizing optical microscope studies revealed that the polymeric gel underwent a reversible order-disorder transition upon an in-

\footnotetext{
* To whom correspondence should be addressed.

+ Present address: Department of Applied Chemistry, Faculty of Science and Engineering, Saga University, 1 Honjo-machi, Saga 840, Japan.
}

crease in temperature. However, the aggregation structure of the stretched crystalline polymeric gel and also, the aggregation structure-thermally responsive property relationships of the crystalline polymeric gel have not been extensively clarified yet. Therefore, the purpose of this study is to understand the aggregation structure of the stearyl acrylate side chains in the (SA/AA/MBAA) gel film and the thermo-responsive mechanism of the crystalline gel film and also, to improve the thermoresponsive behaviors on the basis of the aggregation structural control.

In this study, the crosslinked polymeric gels with crystalline side chains were prepared and the thermoresponsive properties of the unstretched and the stretched gel films were investigated on the basis of the temperature dependence of the swelling ratio and the length of the stretched gel film. Thermally induced aggregation structural changes in the unstretched and the stretched gel films were also investigated on the basis of wide-angle $\mathrm{X}$-ray diffraction (WAXD), small-angle X-ray scattering (SAXS), and differential scanning calorimetric (DSC) measurements.

\section{EXPERIMENTAL}

\section{Preparation of the Gel Film}

Figure 1 shows the chemical structures of monomers used for the preparation of the gel film. The (SA AA/MBAA) gel film was prepared by a free radical copolymerization of stearyl acrylate (SA), acrylic acid (AA), and $N, N^{\prime}$-methylenebisacrylamide (MBAA) which was used as a cross-linking agent. ${ }^{13} \mathrm{SA}$ and AA were dissolved in ethanol $\left(3 \mathrm{~mol} \cdot 1^{-1}\right)$ and then, MBAA, $2,2^{\prime}$ azobis(isobutyronitrile) (AIBN) as an initiator $(1 \mathrm{~mol} \%$ of the monomer charge) were added into a mono- 


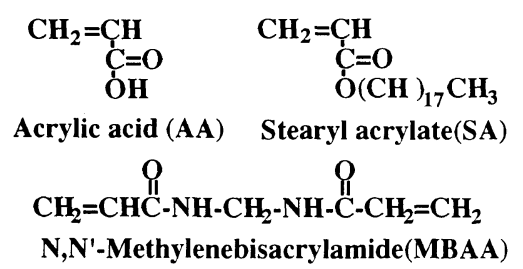

Figure 1. Chemical structures of monomers

mer solution. The monomer solution was degassed in order to reduce the dissolving oxygen. In order to prepare the gel film, the solution of SA, AA, MBAA, and AIBN was placed in a flat petri dish and reacted at $323 \mathrm{~K}$ for $16 \mathrm{~h}$ in a nitrogen atmosphere. In order to remove unreacted monomers, the cross-linked copolymer was equilibrated in dimethyl sulfoxide (DMSO) at $323 \mathrm{~K}$ for a week and dried in vacuo.

\section{Preparation of the Stretched Gel Film}

In order to improve the thermo-responsive behavior, the stretched gel film was prepared by an uniaxial stretching. The swollen (SA/AA/MBAA, 24.7/74.3/1.0; molar ratio) gel film in DMSO was heated up to $328 \mathrm{~K}$ above the melting temperature of stearyl acrylate side chain crystals $\left(T_{\mathrm{m}}=319.3 \mathrm{~K}\right)$. Then, the elastic gel film was uniaxially stretched up to the stretched ratio of 2.5 and 4.0 at $328 \mathrm{~K}$. The stretched gel films were cooled down to room temperature with a given fixed length. The stretching ratio, $\lambda$ was defined as the elongation ratio, that is, ratio of the lengths of the gel film after and before stretching.

\section{Characterizations of the Unstretched and Stretched Gel Films}

Swelling experiments were carried out in DMSO at various temperatures. The (SA/AA/MBAA, 24.7/74.3/ 1.0 ; molar ratio) gel film was used in this study, since this gel film exhibited a crystalline state in a dried state and a swollen one and also, the apparent temperature dependence of the swelling ratio. The gel film was dried in vacuo and weighed to determine the net weight, $W_{0}$. The dried gel film was swollen in DMSO at a given temperature for $48 \mathrm{~h}$ and weighed to determine the equilibrium weight, $W$. The weight swelling ratio was defined as the ratio of $W / W_{0}$.

Thermal properties of the unstretched and the stretched gel films in a dried state and a swollen one were measured by a differential scanning calorimeter (DSC 8230, Rigaku Co., Ltd.) at the heating rate of $1 \mathrm{~K} \cdot \min ^{-1}$ under a dried nitrogen purge.

In order to understand the thermo-responsive mechanism of the polymeric gel and also, to analyze the temperature dependence of aggregation structure change for the unstretched and the stretched gel films, their WAXD and SAXS patterns were taken by using an Imaging Plate System (Mac Science, DIP2000). X-Ray beams were irradiated along the normal (through view, TV), parallel (edge view, EV) to the gel film surface, and parallel to the stretching direction (end view, End).

Thermo-responsive properties was characterized on the basis of the temperature dependence of the length for the stretched gel swollen in DMSO at the heating rate of $1 \mathrm{~K} \cdot \min ^{-1}$.

Polym. J., Vol. 28, No. 5, 1996

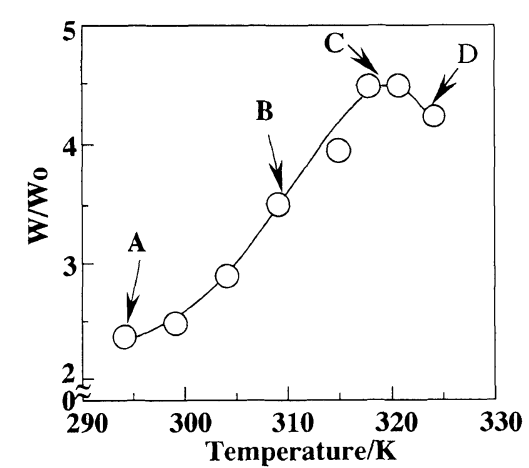

Figure 2. Temperature dependence of the swelling ratio of the (SA AA/MBAA, 24.7/74.3/1.0) gel films containing crystal regions of the SA part in DMSO (A, the room temperature; $R T ; B$, above $R T$ and below the melting temperature, $T_{\mathrm{m}} ; C, T_{\mathrm{m}} ; D$, above $T_{\mathrm{m}}$ ).

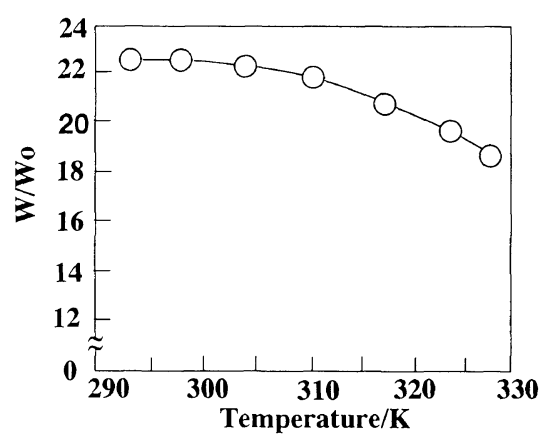

Figure 3. Temperature dependence of the swelling ratio of the (SA/ AA/MBAA, 9.8/89.2/1.0) gel films in an amorphous state in DMSO.

\section{RESULTS AND DISCUSSION}

\section{Temperature Dependence of Weight Swelling Ratio and Aggregation Structure Relationships for Unstretched Gel Film}

Figure 2 shows the temperature dependence of the weight swelling ratio in DMSO for the (SA/AA/MBAA, 24.7/74.3/1.0) gel film containing the crystal region of SA side chains. The weight swelling ratio, $W / W_{0}$ of the gel film in DMSO increased with temperature up to $320 \mathrm{~K}$ and then, decreased above $320 \mathrm{~K}$. In order to investigate the role of the crystalline region on the swelling behavior of the gel film, the temperature dependence of the swelling ratio of the gel film in an amorphous state was measured. Figure 3 shows the temperature dependence of the swelling ratio for the (SA/AA/MBAA, 9.8/89.2/1.0) gel film in an amorphous state in DMSO. The swelling ratio of this gel film decreased with an increase in temperature. Here, it was found from Figures 2 and 3 that the swelling ratio of the (SA/AA/MBAA) gel films decreased with increasing $\mathrm{SA}$ content. This is obviously due to a decrease in the fraction of hydrophilic carboxyl groups of AA and extensive aggregation of hydrophobic long alkyl side groups in this copolymeric gel film. Therefore, it is apparent from Figures 2 and 3 that the temperature dependence of the swelling ratio for the (SA/AA/MBAA) gel film swollen in DMSO strongly depends on the aggregation structure and the molecular mobility of the SA part, in other words, the degrees of crystallinity.

The WAXD and SAXS were measured in order to clarify the aggregation structure for the (SA/AA/MBAA, 24.7/74.3/1) gel film. Figures 4 and 5 show the $X$-ray

Polym. J., Vol. 28, No. 5,1996 


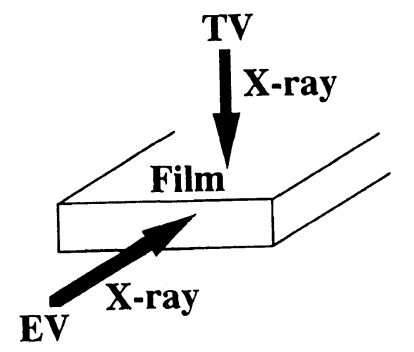

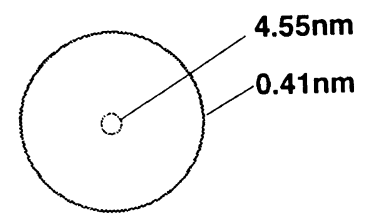

(TV)

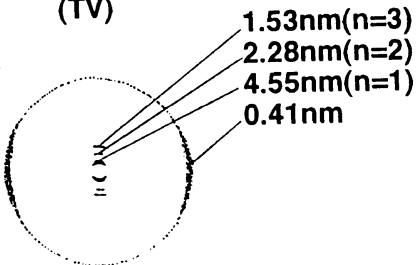

(EV)

(294K)

below $\mathbf{T}_{\mathrm{m}}$

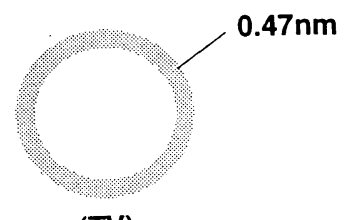

(TV)

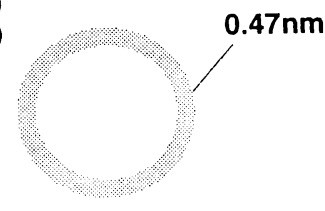

(EV)

(326K)
Figure 4. Schematic representation of X-ray diffraction pattern of the (SA/AA/MBAA, 24.7/74.3/1.0) gel film in a dried state.

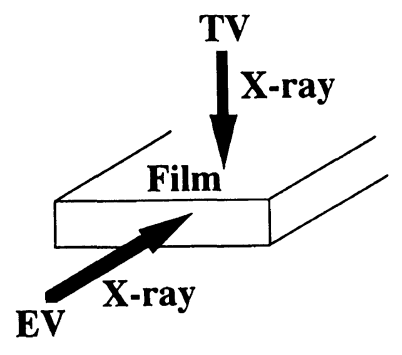

A
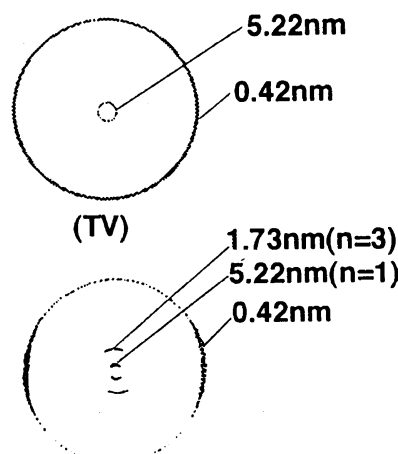

(EV)

(294K)

below $\mathbf{T}_{\mathrm{m}}$

\section{D}

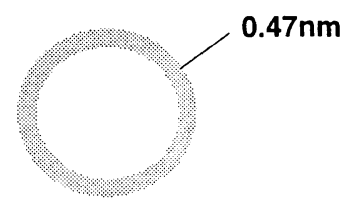

(TV)

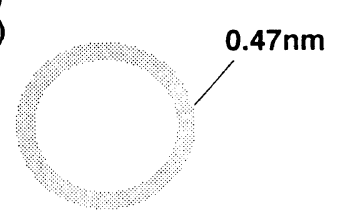

(EV)

(326K) above $\mathbf{T}_{\mathrm{m}}$

Figure 5. Schematic representation of X-ray difraction pattern of the (SA/AA/MBAA, 24.7/74.3/1.0) gel film in a swollen state (A and D region correspond to the temperature region in Figure 2). diffraction patterns of the unstretched (SA/AA/MBAA, 24.7/74.3/1.0) gel film in a dried state and the same gel film in a swollen state $\left(W / W_{0}=2.6\right)$ at $294 \mathrm{~K}$ and $326 \mathrm{~K}$, respectively. When the X-ray beam was irradiated parallel to the film surface (EV), the diffractions with the spacings of $4.55,2.28$, and $1.53 \mathrm{~nm}$ in a dried state and also, 5.22 and $1.73 \mathrm{~nm}$ in a swollen one were observed as arcs on the meridian. Since the reciprocal ratios of these spacings are $1: 2: 3$ in a dried state and $1: 3$ in a swollen one, it may be concluded that SA side chains in the gel film form the bimolecular lamellae or layer structure at $294 \mathrm{~K}$ below the melting temperature of side chain crystals $\left(T_{\mathrm{m}}\right)$. Therefore, both the orientation direction of alkyl chains and the direction of X-ray intensity maxima in the small angle region indicate that the lamellar layer surface align parallel to the film surface and the alkyl chains orient perpendicular to the lamellar. Similar aggregation structure for surface enrichment of the component with lower surface free energy has been observed for the polymer/(fluorocarbon amphiphile) composite thin film. ${ }^{14,15}$ Since the spacings of $0.41 \mathrm{~nm}$ in a dried state and $0.42 \mathrm{~nm}$ in a swollen one attributed to the interchain distance among alkyl groups of the stearyl acrylate side chains were observed intensively on the equator and also, the interchain distances in both a dry state and a swollen one were almost same, it is apparent that DMSO solvent molecules can not penetrate into the side chain crystalline region in the gel film below $T_{\mathrm{m}}$. When the X-ray beam was irradiated perpendicular to the film surface (TV), the crystalline Debye rings corresponding to the spacing of $0.41 \mathrm{~nm}$ in a dried state and $0.42 \mathrm{~nm}$ in a swollen one were observed, respectively. Since the intensity of TV $\mathrm{X}$-ray diffraction is only $1 / 40$ of that in EV, it seems reasonable to consider that lamellae composed of SA alkyl side chains are randomly oriented along the axis perpendicular to the film surface. Then, it can be concluded from the results of X-ray diffraction and DSC studies that alkyl side chains in the gel film form the crystalline lamellae below $T_{\mathrm{m}}$ both in a dried state and a swollen one. On the other hand, since only the amorphous halo was observed at $326 \mathrm{~K}$ in a dried state or a swollen one, it is apparent that the crystalline lamellae were disappeared owing to melting of alkyl side chain crystals in the gel film.

Further, one should notice here that the lamellar long period $(4.55 \mathrm{~nm})$ in the gel film is shorter than twice of SA side chain length $(5.16 \mathrm{~nm})$ in assumption that the alkyl chain is in an all-trans conformation. Figure 4 clearly shows the perpendicular orientation of SA alkyl side chains to the lamellar surface. Then, there are two possibilities to explain the difference between the experimental lamellar long period and twice of the calculated SA alkyl side chain length in a trans conformation: the interdigitated structure for the side chain molecules or the introduction of gauche conformation around at the alkyl chain end and/or the connecting portion to the main chain, because the oblique structure for the side chain molecules was not confirmed from the WAXD pattern. At present time, the conclusion has not been obtained yet. However, if the crystalline structure for the side chain molecules forms the interdigitated structure, such a structure will be energetically un- 


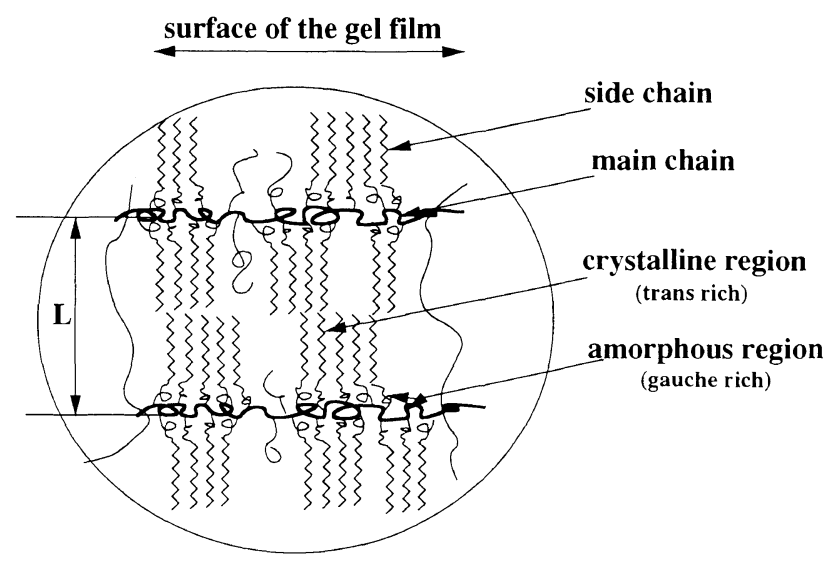

Figure 6. Schematic representation of aggregation structure of the long side chains for the dried (SA/AA/MBAA, 24.7/74.3/1.0) gel film.

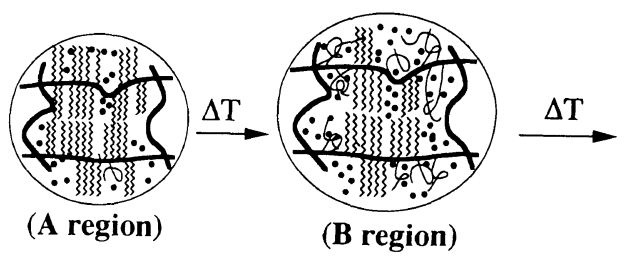

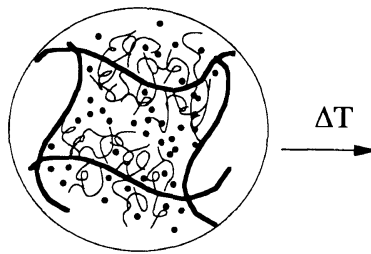

(C region)

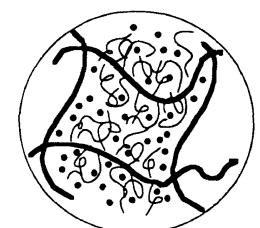

(D region)
Figure 7. Schematic representation of swelling behavior of the (SA/ AA/MBAA, 24.7/74.3/1.0) gel film swollen in DMSO (A, B, C, and $\mathrm{D}$ region correspond to the temperature region in Figure 2).

favorable because there are only about five $\mathrm{CH}_{2}$ groups interdigitated. Therefore, the schematic representation of a proposed aggregation structure of SA side chains for the dried (SA/AA/MBAA, 24.7/74.3/1.0) gel film shown in Figure 6 was drawn on the basis of partial introduction of gauche chains.

Figure 7 shows the schematic representation of thermally-induced aggregation structural change of the (SA/ AA/MBAA, 24.7/74.3/1.0) gel film swollen in DMSO. These aggregation structure changes were concluded on the basis of the DSC, WAXD, and SAXS results as mentioned above. The swelling ratio of the gel film in DMSO strongly depends on the network structure, temperature, and the polymer-solvent interaction parameter. A, B, C, and D regions in Figure 7 correspond to each temperature were indicated in Figure 2. In the case of the (SA/AA/MBAA) gel film, the swelling ratio swollen in DMSO increased with temperature up to the $T_{\mathrm{m}}$ of the SA alkyl chain as shown in Figure 2 and Figure 7, because DMSO molecules may more easily penetrate into the amorphous region or the AA-rich region and also, an apparent amorphous fraction may increase with temperature owing to the partial melting of the SA side chain crystal. On the other hand, in a temperature range above the melting temperature of SA side chain crystal, the swelling ratio decreased with an increase in temperature owing to thermal shrinkage of a main chain caused by rubber elasticity characteristics of the network

Polym. J., Vol. 28, No. 5, 1996

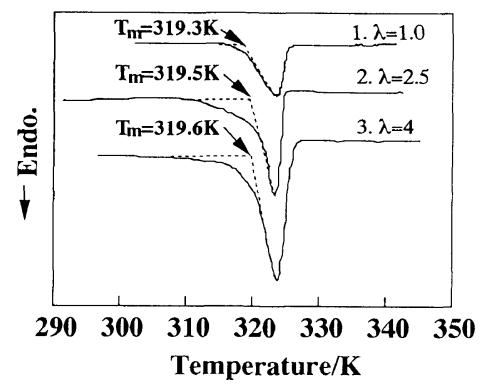

Figure 8. DSC thermograms of the stretched (SA/AA/MBAA, 24.7/ $74.3 / 1.0)$ gel films in DMSO.

structure in the gel film. Therefore, the swelling ratio showed a maxima when the crystalline region almost completely melted as shown in Figure 2.

\section{Structural Characterizations and Thermo-Responsive Prop- erties of the Stretched Gel Film}

In order to improve the thermo-responsive behavior, the stretched gel film was prepared by an uniaxial stretching. Thermal properties and thermally-induced aggregation structural change for the stretched gel films were investigated on the basis of DSC and X-ray diffraction measurements.

Figure 8 shows the DSC thermograms for the stretched (SA/AA/MBAA, 24.7/74.3/1.0) gel films swollen in DMSO. The magnitude of melting endotherm were $15.6 \mathrm{~kJ}(\mathrm{SA} \mathrm{mol})^{-1}(\lambda=1.0), 19.8 \mathrm{~kJ}(\mathrm{SA} \mathrm{mol})^{-1}(\lambda=2.5)$, and $22.7 \mathrm{~kJ}(\mathrm{SA} \mathrm{mol})^{-1}(\lambda=4.0)$. Since the magnitude of melting endotherm increased with the stretching ratio, it is apparently concluded that the crystallinity and/or the regularity of layer structure in the stretched gel film was improved by stretching.

Figure 9 shows the X-ray diffraction patterns for the stretched (SA/AA/MBAA) gel film in DMSO $\left(W / W_{0}=\right.$ $2.1)$ at $294 \mathrm{~K}$ and $326 \mathrm{~K}$. When the $\mathrm{X}$-ray beam was irradiated perpendicular to the stretching direction (EV and TV), the X-ray scattering arcs with the spacings of 5.28 and $1.78 \mathrm{~nm}$ were observed perpendicular to the stretching direction. The crystalline arc with the spacing of $0.42 \mathrm{~nm}$ corresponding to the intermolecular distance among SA alkyl side chains was also observed parallel to the stretching direction at $294 \mathrm{~K}$. Small angle reflections in the stretched gel film were shaper in comparison with those of the unstretched gel film. These results indicated that the regularity of layer structure was improved by uniaxial stretching. The result of X-ray diffraction measurement agrees fairly well with the result from DSC. When the X-ray beam was irradiated parallel to the stretching direction (End View), Debye rings at small and wide angle regions were observed as shown in the lower part of Figure 9. Then, it is apparent that bimolecular lamellar crystals in the stretched gel film form uniaxial orientation, i.e., the lamellar long axis aligned along the stretching direction and the molecular axes of SA side chains in the lamellae oriented perpendicular to the stretching direction, with random orientation around the stretching axis. Further, amorphous halo was observed in the stretched gel film at $326 \mathrm{~K}$, owing to the melting of side chain crystals.

Figure 10 shows the schematic representation of the lamellar orientation in the stretched (SA/AA/MBAA) gel 

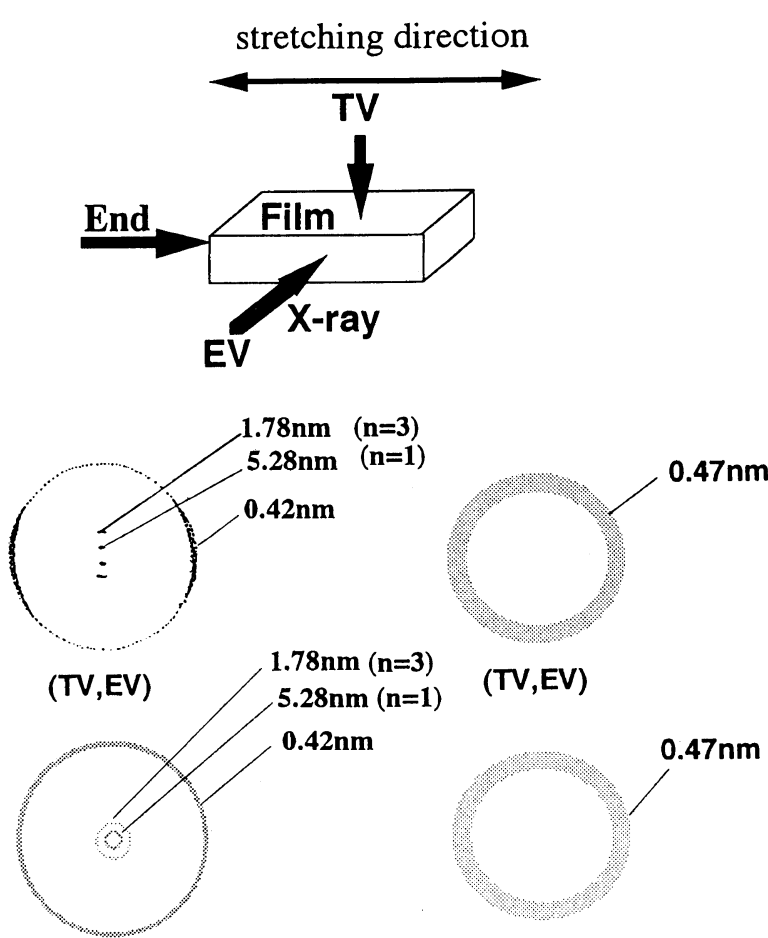

(End)

(294K)
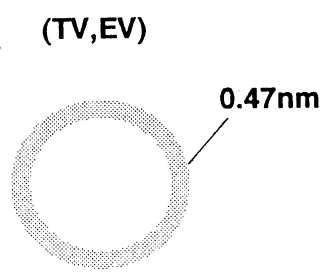

(End)

(326K)

below $\mathbf{T}_{\mathrm{m}}$

above $\mathbf{T}_{\mathrm{m}}$

Figure 9. Schematic representation of X-ray difraction patterns of the stretched (SA/AA/MBAA, 24.7/74.3/1.0) gel film $(\lambda=4)$ in DMSO.

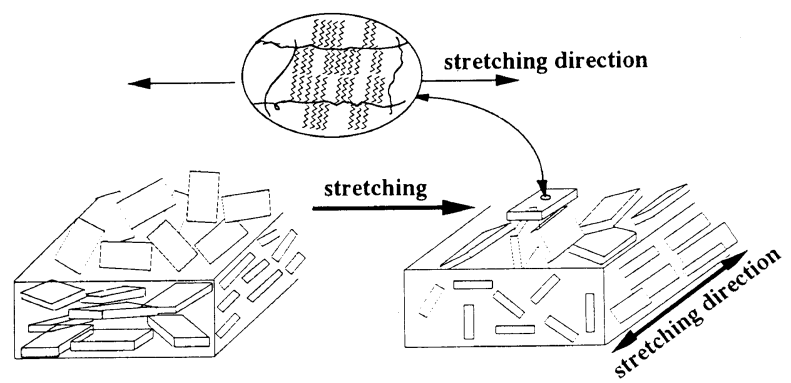

Figure 10. Model of aggregation state change of the (SA/AA/MBAA, $24.7 / 74.3 / 1.0)$ gel film upon stretching.

film. Although the spacings of the interchain distance among alkyl side chains of SA and the long periods of the lamellae in the unstretched and the stretched gel films were almost the same, the rearrangement of the lamellar assembly was occurred during stretching. The lamellae in the unstretched gel were randomly orientated along the surface of the gel film swollen in DMSO, that is, uniplanar orientation. The lamellar long axes in the stretched gel oriented parallel to the stretching direction with a cylindrically symmetrical orientation. The molecular axes of stearyl acrylate side chains were maintained perpendicular orientation to the stretching direction during stretching. Also, from the DSC, WAXD, and SAXS results as mentioned above, it is apparent that the crystallinity and/or the regularity of layer structure in the stretched gel film was increased by uniaxial stretching.

The degree of crystallinity and/or the regularity of layer structure in the stretched gel film was increased by uniaxial stretching, as mentioned above. In order to

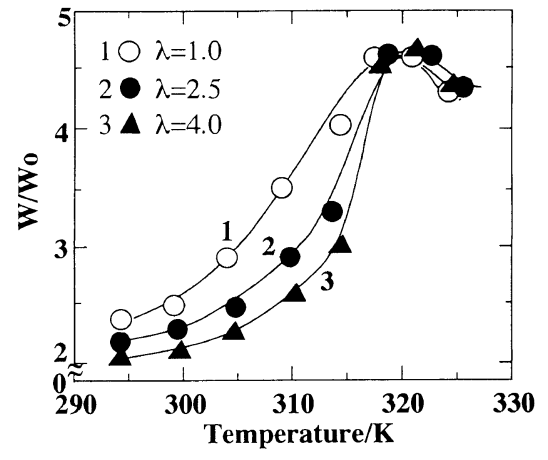

Figure 11. Temperature dependence of the swelling ratio for the stretched (SA/AA/MBAA, 24.7/74.3/1.0) gel films in DMSO.

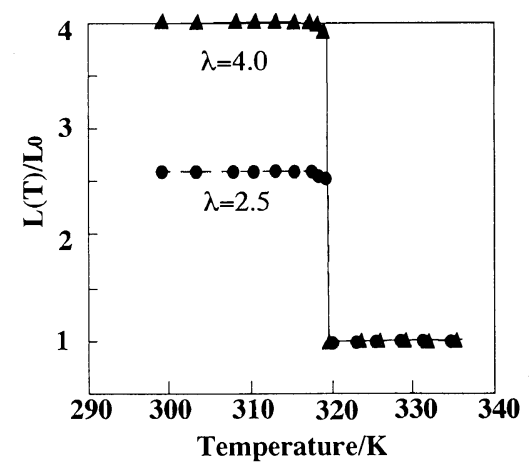

Figure 12. Temperature dependence of specimen length of the stretched (SA/AA/MBAA, 24.7/74.3/1.0) gel films swollen in DMSO.

observe the difference in the thermo-responsive properties of the stretched gel from that of the unstretched gel, the temperature dependence in the swelling ratio and in the length of the stretched gel were investigated. Figure 11 shows the temperature dependence of the swelling ratio for the stretched (SA/AA/MBAA, 24.7/74.3/1.0) gel film swollen in DMSO. The temperature dependence of swelling ratio for the stretched gel films became more distinctly with an increase in stretching ratio. As mentioned above, DMSO molecules can not penetrate into the side chain crystalline region in the gel film at room temperature. Also, the stretched gel film showed a decrease in the swelling ratio because the crystallinity and/ or the regularity of layer structure for the stretched gel films were increased with the stretching, as mentioned above. As shown in Figures 2 and 11, the swelling ratios of the stretched and the unstretched gel film were almost the same when the crystalline region in the stretched gel films completely melted above the melting temperature of the side chain crystals. Therefore, it seems reasonable to conclude that the thermo-responsive behavior for the stretched gel films might be strongly reflected the structurally controllable factors such as orientation and crystallization of SA side chains.

Figure 12 shows the temperature dependence of the specimen length ratio, $L(T) / L_{0}$ for the stretched gel film without load. $L_{0}$ and $L(T)$ are the length for the gel film before and after stretching at a given temperature, respectively. When the stretched gel film was heated above $T_{\mathrm{m}}$ of SA side chain crystals, the stretched gel abruptly shrinked to the original length, $L_{0}$. This behavior was induced by rubber elasticity characteristics of the network structure in the stretched crystalline gel 


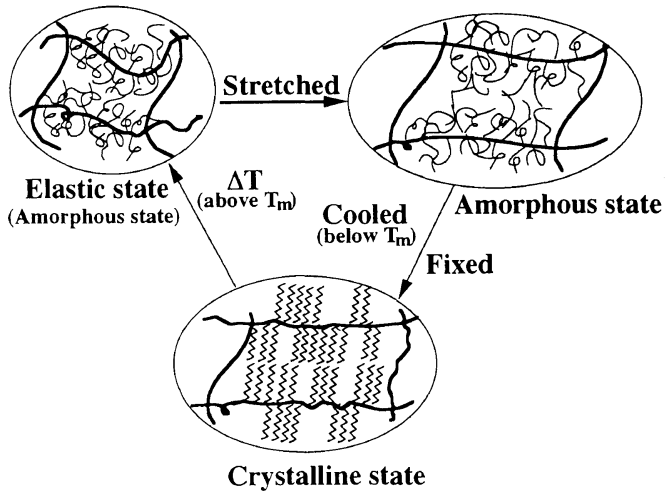

Figure 13. Model of phase change behaviors of the stretched (SA/ AA/MBAA, 24.7/74.3/1.0) gel film in DMSO.

film that was induced by the melting of side chain crystals. The responsive speed for shrinking increased with the stretching ratios. The result of Figure 12 indicates that the shape memory effect of the crystalline (SA/AA/MBAA) gel film was realized by uniaxial stretching due to the orientation crystalline lamellae composed of side chain crystals. Figure 13 shows the schematic representation of the phase transition behaviors and the molecular aggregation structure change with temperature and elongation for the stretched gel film in DMSO. The shape of the stretched gel film is stable below $T_{\mathrm{m}}$ of SA side chain crystals, because the stretched gel film is composed of the oriented lamellar crystal with the fairly strong intermolecular aggregation structure of side chains. When the side chain crystals in the stretched gel were melted at $T_{\mathrm{m}}$, the stretched gel films shrinked back to the original length owing to its rubber elasticity characteristics of the network structure in the stretched gel film.

\section{CONCLUSION}

The (SA/AA/MBAA, 24.7/74.3/1.0; molar ratio) gel film forms crystalline bimolecular lamellar structure owing to intermolecular aggregation of stearyl acrylate side chains in both a dried state and in a swelling one at room temperature. The model of the SA side chains aggregation structure for this dried gel film was proposed, i.e., the introduction of gauche conformation around at the alkyl chain end and/or the connecting portion to the main chain. Also, the bimolecular lamellar layer surface in the unstreched gel film align parallel to the film surface and the alkyl chains orient perpendicular to the lamellar. The thermally induced responsive behaviors of the (SA/ AA/MBAA) gel film in DMSO is strongly related to the aggregation structure and the molecular mobility of stearyl acrylate side chain groups. The swelling ratio of the gel film in DMSO increased with temperature up to the melting temperature of stearyl side groups and decreased above the melting temperature. The molecular axes of stearyl side groups in the stretched gel were maintained perpendicular orientation to the stretching direction during stretching, i.e., the bimolecular lamellae long axes oriented parallel to the stretching direction with a cylindrically symmetrical orientation and also, the crystallinity and/or the regularity of layer structure in the stretched gel film was increased by stretching. The thermo-responsive behavior for the stretched gel films became more distinct with the increase in crystallinity due to the uniaxial elongation. The stretched gel film showed the irreversible shape memory effect due to its rubber elasticity characteristics being induced by the melting of the crystalline lamellar structure composed of the stearyl acrylate side chains.

\section{REFERENCES}

1. Y. Osada, Adv. Polym. Sci., 82, 1 (1987)

2. T. Okano, Adv. Polym. Sci., 110, 179 (1993).

3. S. Katayama, Y. Hirokawa, and T. Tanaka, Macromolecules, 17, 2641 (1984).

4. F. S. Freitas and E. L. Cussler, Chem. Eng. Sci., 42, 97 (1987)

5. L. D. Taylor and L. D. Cerankowki, J. Polym. Sci., Polym. Chem. Ed., 13, 2551 (1984).

6. A. H. Clark and S. B. Ross-Murphy, Adv. Polym. Sci., 83, 57 (1987).

7. K. Samejima, Y. Hashimoto, T. Yasui, and T. Fukuzawa, J. Food Sci., 34, 242 (1969).

8. T. Mitsui and H. Ohshima, J. Muscle Res. Cell Motility, 9, 248 (1988).

9. T. Tanaka, Sci. Am., 249, 124 (1981).

10. S. H. Gehrke, Adv. Polym. Sci., 110, 81 (1993).

11. Y. Osada and H. Yasunaga, Kobunshi Ronbunshu, 46, 655 (1989).

12. A. Matsuda, J. Sato, H. Yasunaga, and Y. Osada, Macromolecules, 27, 7695 (1994)

13. X. W. He, Y. Oishi, A. Takahara, and T. Kajiyama, Rep. Prog. Polym. Phys. Jpn., 37, 129 (1994).

14. A. Takahara, N. Higashi, T. Kunitake, and T. Kajiyama, Macromolecules, 21, 2443 (1988).

15. A. Takahara, N. Morotomi, S. Hiraoka, N. Higashi, T. Kunitake, and T. Kajiyama, Macromolecules, 22, 617 (1989). 